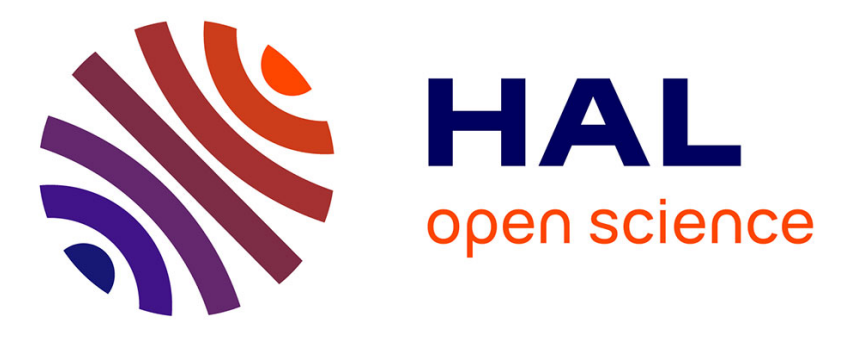

\title{
Thermal interfacial transport in the presence of ballistic heat modes
}

\author{
Bjorn Vermeersch, Amr M. S. Mohammed, Gilles Pernot, Yee Rui Koh, Ali \\ Shakouri
}

\section{- To cite this version:}

Bjorn Vermeersch, Amr M. S. Mohammed, Gilles Pernot, Yee Rui Koh, Ali Shakouri. Thermal interfacial transport in the presence of ballistic heat modes. Physical Review B: Condensed Matter and Materials Physics (1998-2015), 2014, 90 (1), pp.014306. 10.1103/PhysRevB.90.014306 . hal01063535

\section{HAL Id: hal-01063535 \\ https://hal.science/hal-01063535}

Submitted on 23 Feb 2016

HAL is a multi-disciplinary open access archive for the deposit and dissemination of scientific research documents, whether they are published or not. The documents may come from teaching and research institutions in France or abroad, or from public or private research centers.
L'archive ouverte pluridisciplinaire HAL, est destinée au dépôt et à la diffusion de documents scientifiques de niveau recherche, publiés ou non, émanant des établissements d'enseignement et de recherche français ou étrangers, des laboratoires publics ou privés. 


\title{
Thermal interfacial transport in the presence of ballistic heat modes
}

\author{
Bjorn Vermeersch, ${ }^{1, *}$ Amr M. S. Mohammed, ${ }^{1, \dagger}$ Gilles Pernot, ${ }^{2}$ Yee Rui Koh, ${ }^{1}$ and Ali Shakouri ${ }^{1, \dagger}$ \\ ${ }^{1}$ Birck Nanotechnology Center, Purdue University, West Lafayette, Indiana 47907, USA \\ ${ }^{2}$ LOMA, Université de Bordeaux 1, 33400 Talence, France
}

(Received 2 February 2014; revised manuscript received 8 July 2014; published 25 July 2014)

\begin{abstract}
Thermal interface (Kapitza) resistance expresses how hard it is for heat to flow across material junctions inside multilayer structures. This quantity plays a crucial role in the thermal performance of nanoscale devices but is still poorly understood. Here we show that conventional Fourier-based metrology overestimates metal/semiconductor resistances by up to threefold due to misinterpretation of ballistic heat flow modes. We achieve improved identification and a different physical insight with a truncated Lévy formalism. This approach properly distinguishes interfacial dynamics from nearby quasiballistic heat flow suppression in the semiconductor. Unlike conventionally extracted values, interface resistances obtained with our new approach are independent of laser modulation frequency, as physically appropriate, and much more closely approach theoretical predictions.
\end{abstract}

PACS number(s): $63.20 . \mathrm{kg}, 65.40 .-\mathrm{b}, 66.70 .-\mathrm{f}$

Despite seven decades of extensive research since Kapitza's pioneering work [1], a comprehensive understanding of heat flow across material interfaces has remained a mostly open problem [2]. Acoustic mismatch (AMM) and diffusive mismatch (DMM) models $[3,4]$ have made important progress but none of the currently available theories can fully describe measured thermal interface resistances for diverse metals, dielectrics, and semiconductors. As miniaturization of semiconductor technologies is continuously pushed to further extremes, the thermal performance of electronics and optoelectronics is dominated more and more by interface effects $[5,6]$. Improving the metrology and conceptual understanding of Kapitza resistance is therefore not only of fundamental interest for the thermal physics and material science communities, but also highly relevant to a wide array of nanoscale device applications.

Metal/semiconductor contacts are thermally evaluated by means of their interface resistivity $r_{\mathrm{ms}}\left(\mathrm{nK}-\mathrm{m}^{2} / \mathrm{W}\right)$ or associated conductance $G_{\mathrm{ms}}=r_{\mathrm{ms}}^{-1}\left(\mathrm{GW} / \mathrm{m}^{2}-\mathrm{K}\right)$. In the chosen units, $r_{\mathrm{ms}}$ values correspond to the thickness in nanometers of a material with unit thermal conductivity that would be needed to induce the same temperature drop in the heat flow path as that observed across the interface.

Experimental characterization of $r_{\mathrm{ms}}$ is usually carried out by fitting measured thermal responses of metal-onsemiconductor structures to a conventional Fourier diffusion model. Most experiments subject the test sample to ultrashort energy impulses and observe only the first couple of nanoseconds of the induced temperature decay [7-9]. The heat flow across and near the metal/semiconductor interface is therefore highly dynamic, warranting caution on how to extract information from the captured transients. A variety of studies have demonstrated that short-time and short-lengthscale thermal transport in semiconductors exhibits notable quasiballistic effects [10-13]. In this work, we demonstrate

\footnotetext{
*These authors contributed equally to this work: bvermeer@ purdue.edu

${ }^{\dagger}$ These authors contributed equally to this work: amrmoham@ purdue.edu

${ }^{\ddagger}$ shakouri@purdue.edu
}

that conventional Fourier-based models produce a severe nonphysical dependence of $r_{\mathrm{ms}}$ on laser modulation frequency when quasiballistic effects are present in the semiconductor. This problem disappears, and the overall fitting quality improves, when we instead use a truncated Lévy (TL) heat formalism which describes the quasiballistic heat flow in terms of non-Brownian transport dynamics. Our findings suggest that this novel approach is much better suited for characterization of the intrinsic performance of the thermal interface. Metal/semiconductor interfaces are found to be up to three times more conductive than currently believed, closing most of the gap that thus far had remained between experimental values and theoretical predictions.

State-of-the-art characterization of interfacial thermal transport typically relies on transient laser thermoreflectometry [7-9]. A "pump" beam, modulated at frequency $f_{\text {mod }}$, heats the sample while lock-in detection of the "probe" beam reflection captures the thermal response as a function of the pump-probe delay. A 50- to $100-\mathrm{nm}$ thin layer of the metal is deposited on top of the semiconductor surface to act as measurement transducer. Fitting the measured thermal decay of the transducer surface to a Fourier diffusion model provides the metal/semiconductor interface resistivity $r_{\mathrm{ms}}$ and semiconductor thermal conductivity $k[10,14]$. Results of this conventional identification obtained for an $\mathrm{Al} / \mathrm{InGaAs}$ sample are shown in Fig. 1.

Remarkably, the apparent semiconductor conductivity drops by more than $40 \%$ over the 0.8 - to $20-\mathrm{MHz}$ pump modulation frequency range [Fig. 1(a)]. This anomalous behavior, first pointed out by Koh and Cahill [10] and subsequently confirmed by independent studies $[11,13,15]$, is attributed to quasiballistic heat flow. Phonon modes with mean free paths (MFPs) longer than the thermal penetration length of the experiment, $\ell=\left[D /\left(\pi f_{\bmod }\right)\right]^{1 / 2}$, with $D$ the semiconductor diffusivity, are thought not to contribute to the "apparent" thermal conductivity [10,11]. First-principles calculations show that even at room temperature, a significant fraction of thermal transport in semiconductors is governed by phonons with MFPs exceeding 1-10 $\mu \mathrm{m}[16,17]$. As transient laser experiments readily achieve penetration lengths $\ell \leqslant 300 \mathrm{~nm}$ in typical semiconductor alloys, we can expect quasiballistic heat modes to notably influence thermal characterization. 

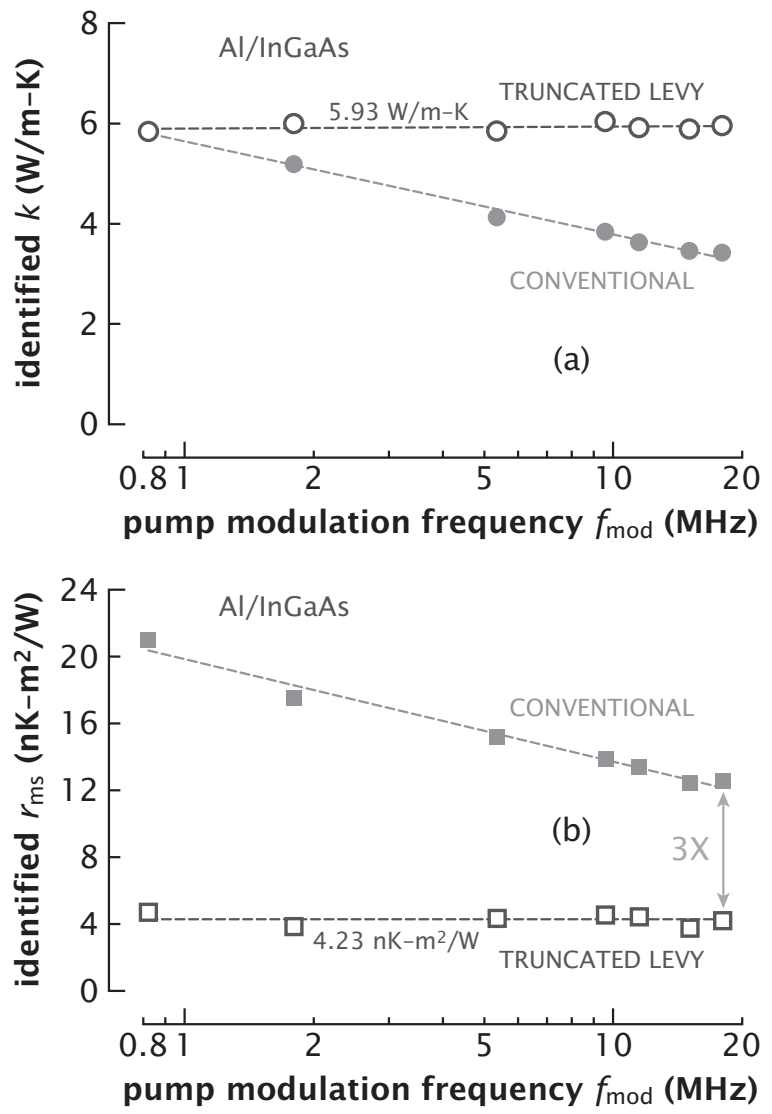

FIG. 1. Time-domain thermoreflectance characterization of $\mathrm{Al} / \mathrm{InGaAs}$ sample at room temperature. A theoretical model is fitted to lock-in measurements of the thermal transient of the metal transducer surface at laser modulation frequency $f_{\text {mod }}$. This provides the thermal resitivity $r_{\mathrm{ms}}$ of the metal/semiconductor interface and thermal conductivity $k$ of the semiconductor. Conventional approaches use a regular Fourier diffusion model and perceive "effective" parameters that strongly vary with $f_{\text {mod }}$ due to quasiballistic effects. The truncated Lévy approach describes the quasiballistic transport as a non-Brownian superdiffusive process and extracts "intrinsic" parameters that are independent of frequency as physically appropriate.

While a variable effective conductivity $k_{\text {eff }}\left(f_{\text {mod }}\right)$ can be given at least some physical interpretation as sketched above, the strong dependence of Fourier-identified interface resistivity on modulation frequency [Fig. 1(b)] is far more problematic. Even at $20 \mathrm{MHz}$, the characteristic time constant associated with the oscillating heat source, $\tau_{\text {mod }}=$ $\left(2 \pi f_{\text {mod }}\right)^{-1} \approx 8 \mathrm{~ns}$, is still several orders of magnitudes larger than the typical electron-phonon and phonon-phonon scattering rates [18]. The dynamic processes governing the interfacial thermal transport are therefore capable of following the sinusoidal variation of the incident heat flux instantaneously, implying that $r_{\mathrm{ms}}$ should remain constant over the considered modulation frequency range $[10,13]$.

The apparent variation of interface resistivity with measurement frequency perceived by conventional approaches indicates that Fourier diffusion theory is inadequate to interpret the rich physics of the measured thermal transients. This situation can be resolved by properly distinguishing interface dynamics from nearby quasiballistic thermal transport in the semiconductor. Recently, we have introduced a new TL formalism that incorporates nondiffusive heat components [19]. The core idea is that we put forward a different description of the microscopic random dynamics of the energy carriers inside the semiconductor. This provides a much better representation of the ballistic heat components than can be achieved by adjusting macroscopic bulk parameters with measurement frequency as done in conventional Fourier approaches. Fourier diffusion is governed by regular Brownian motion, in which random walking energy carriers perform consecutive independent jumps with jump length probability $p_{\mathrm{U}}(u) \propto u^{-3}$. Instead, we proposed a TL flight process of the form

$$
p_{\mathrm{U}}(u) \propto \exp \left(-u / u_{\mathrm{BD}}\right) \cdot u^{-(1+\alpha)}, \quad 0<\alpha<2 .
$$

The associated thermal field exhibits a natural transition from a superdiffusive Lévy regime with fractal dimension $\alpha$ [one-dimensional (1D) heat source response $T_{0} \sim t^{-1 / \alpha}$ ] to regular Fourier diffusion $\left(T_{0} \sim t^{-1 / 2}\right)$ over length scales of the order of $u_{\mathrm{BD}}$. Additional details are available elsewhere [19]. Our approach provides an excellent match to raw experimental data over the entire $f_{\text {mod }}$ range with just four parameters [20]. Crucially, processing the earlier mentioned $\mathrm{Al} / \mathrm{InGaAs}$ measurements frequency by frequency with our TL formalism yields $k$ and $r_{\mathrm{ms}}$ values that are virtually independent of pump modulation frequency (Fig. 1), as physically appropriate. Unlike conventional Fourier analyses, the TL formalism is thus capable of distinguishing bulk thermal properties $\left(k, r_{\mathrm{ms}}\right)$ from ballistic effects $\left(\alpha, u_{\mathrm{BD}}\right)$. This decoupled extraction provides additional insights and offers potential for more accurate thermal metrology.

At a low modulation frequency the conventionally extracted effective conductivity, known to compare favorably with $3 \omega$ measurements $[10,21]$, approaches the TL-identified bulk value [Fig. 1(a)]. As the thermal penetration length starts to exceed the majority of the phonon MFPs the transport becomes predominantly diffusive, and we can indeed expect the methods to converge. Such agreement is far from reached with regard to the metal/semiconductor resistance. Even at the highest frequency, where conventional identification is typically performed to exploit the highest sensitivity [13], a mismatch by a factor of 3 is observed [Fig. 1(b)].

We argue that conventional Fourier-based metrology severely overestimates $r_{\mathrm{ms}}$, as it will mistakenly interpret the quasiballistic heat flow suppression in the upper semiconductor region as a poorer interface performance. The effect can be illustrated by considering the calculated interfacial heat flux generated by a single energy impulse on the transducer (Fig. 2). Reverting the energy carrier dynamics from Brownian to TL while maintaining nominal $k$ and $r_{\mathrm{ms}}$ lowers the peak heat flux (curve A to curve B). A similar reduction is observed for a Fourier model with increased interface resistivity (curve C), explaining the tendency for overestimation of $r_{\mathrm{ms}}$ when nondiffusive effects are present. These insights also lend some critical perspective to the common notion of ballistic heat modes simply acting as additional thermal resistance [5,12]. While a Fourier model with adjusted $r_{\mathrm{ms}}$ can achieve the appropriate magnitude of the interfacial heat flux, the temporal and spatial shape of the thermal field still differ notably from 


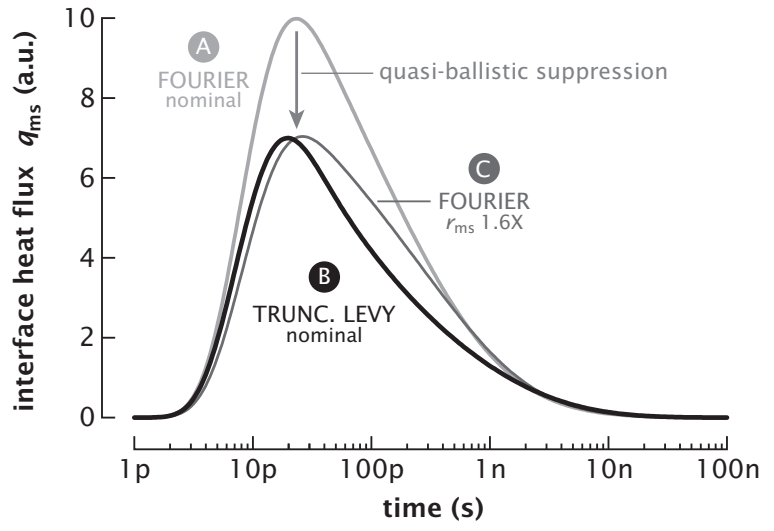

FIG. 2. Conventional metrology overestimates the thermal interface resistance when quasiballistic effects are present. Calculated interfacial heat flux versus time for a single energy impulse at the transducer surface. The truncated Lévy heat flux (curve B) is lower than the nominal Fourier prediction (curve A) due to incorporation of quasiballistic transport modes. A similar suppression can be obtained phenomenologically by a Fourier model with increased $r_{\text {ms }}$ (curve C), illustrating the overestimation tendency.

the TL counterparts [19]. This again highlights the intricate details of the quasiballistic transport dynamics.

The results above show that the TL process induces a heat flux suppression, in accordance with earlier theoretical and experimental analyses of quasiballistic transport [15,22]. At the same time, superdiffusion is commonly associated with improved, not impeded, energy transport [23]. The apparent contradiction can be resolved by carefully considering how improved transport is defined. The effects of the quasiballistic transport modes can be understood by looking at how the TL 1D single pulse response evolves in space and time (Fig. 3).

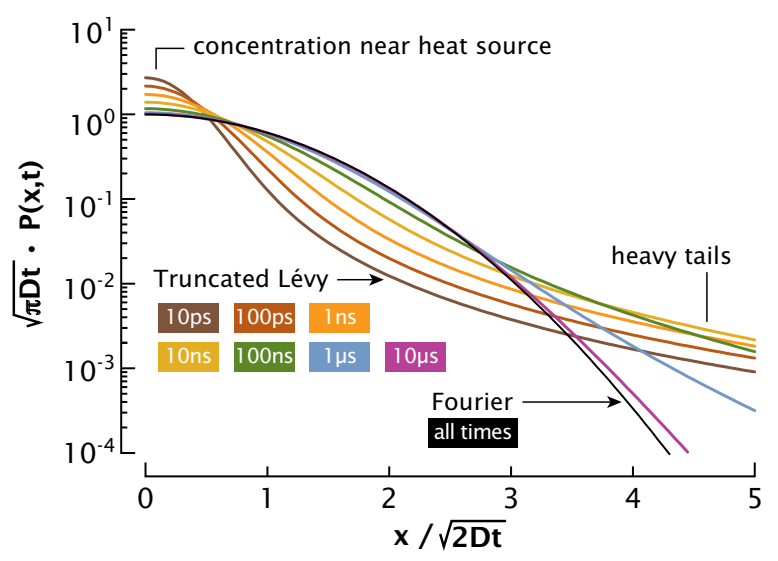

FIG. 3. (Color online) Normalized 1D energy density at several indicated times versus normalized position inside semi-infinite InGaAs for a $1 \mathrm{~J} / \mathrm{m}^{2}$ impulse at the top surface, $x=0$. The Fourier response is Gaussian at all times. During the quasiballistic regime, truncated Lévy profiles show heat concentration near the source, reduced heating at intermediate distances, and "heavy tails" at large distances. As time progresses the transport gradually becomes more and more diffusive, and TL distributions converge to Fourier solutions at times of the order of $t_{\mathrm{BD}}=u_{\mathrm{BD}}^{2} / 2 D$ (a few microseconds in InGaAs).
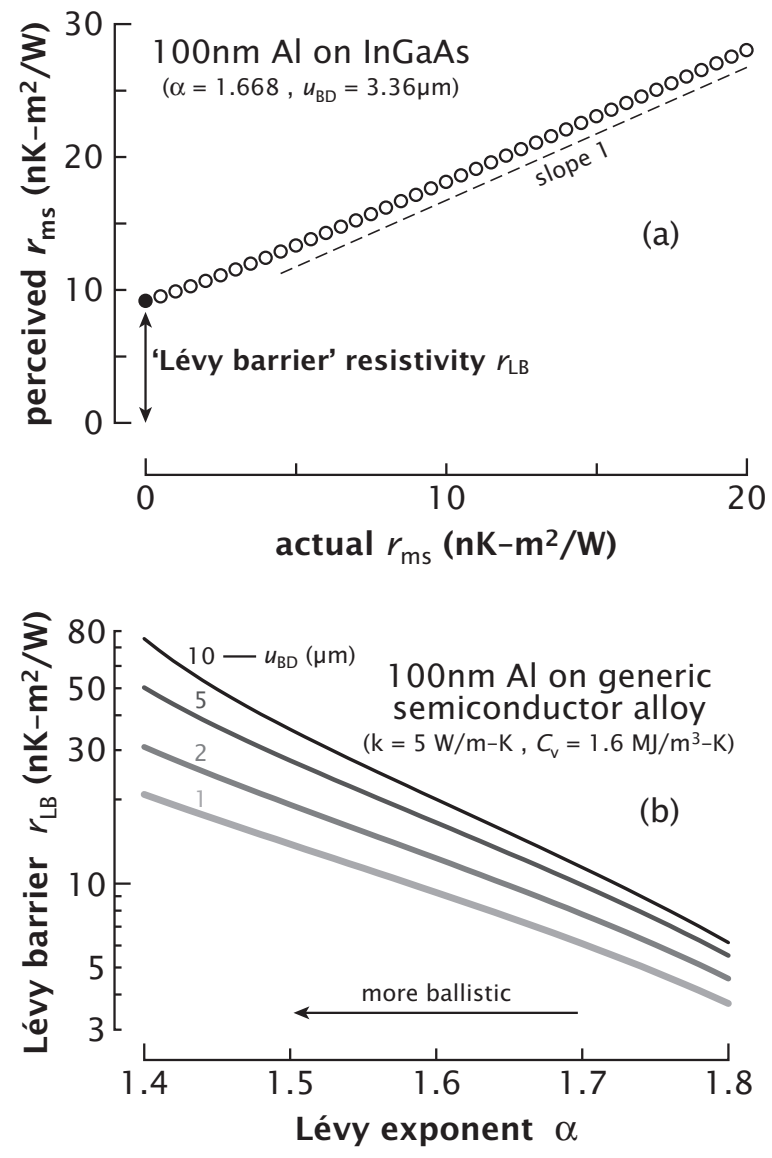

FIG. 4. Lévy barrier in conventional interface metrology. (a) Fourier identification of truncated Lévy simulations. The conventionally perceived interface resistivity is consistently larger than the actual value set in the simulation. Even when a thermally perfect interface is assumed (actual $r_{\mathrm{ms}} \equiv 0$ ), a significant resistivity $r_{\mathrm{LB}}$ is perceived. We call this the "Lévy barrier" resistivity, as this quantity can be directly attributed to Fourier misinterpretation of quasiballistic transport effects. (b) Calculated Lévy barrier resistivity as a function of the ballistic parameters of a generic semiconductor alloy. The barrier becomes larger as the superdiffusive transport deviates more severely from Brownian dynamics (smaller $\alpha$ ) and persists over longer distances (larger $u_{\mathrm{BD}}$ ).

Deep inside the medium, a few penetration lengths $\ell=$ $\sqrt{2 D t}$ away from the heat source, the TL response is notably higher than the nominal Fourier predictions. These "heavy tails," made possible by the more frequent occurrence of long jumps, indicate that superdiffusive modes move some of the energy farther and /or faster into the medium than regular diffusion. This can indeed be interpreted as improved transport. At the same time, the TL response is also several times larger than the Fourier counterpart at and near the heat source. It is this tendency that makes it harder for the energy in the experimental configuration to leave the transducer, leading to the reduction in peak heat flux shown in Fig. 2.

Further evidence that conventional analyses misinterpret ballistic effects as a higher interface resistivity can be found through TL simulations of structures with various $r_{\mathrm{ms}}$ values. Fourier identification of the simulated response yields an interface resistivity that is consistently higher than the actual 
TABLE I. Identified thermal parameters for three material systems at room temperature.

\begin{tabular}{lccc}
\hline \hline & $\mathrm{Al} / \mathrm{InGaAs}$ & $\mathrm{Al} / \mathrm{SiGe}$ & $\mathrm{Al} / \mathrm{GaAs}$ \\
\hline$\alpha$ & 1.668 & 1.690 & 1.565 \\
$u_{\mathrm{BD}}(\mu \mathrm{m})$ & 3.36 & 1.97 & 0.235 \\
$k(\mathrm{~W} / \mathrm{m}-\mathrm{K})$ & & & \\
$\quad$ Conventional & $5.85-3.42$ & $3.88-2.63$ & $40.6-39.2$ \\
$\quad$ Trunc. Lévy & 5.94 & 4.03 & 40.2 \\
$r_{\text {ms }}\left(\mathrm{nK}-\mathrm{m}^{2} / \mathrm{W}\right)$ & & & \\
Conventional & 12.44 & 12.21 & 7.62 \\
$\quad$ Trunc. Lévy & 4.14 & 5.13 & 7.25 \\
Conv. overest. & $3.0 \mathrm{X}$ & $2.4 \mathrm{X}$ & $1.05 \mathrm{X}$ \\
$r_{\mathrm{LB}}\left(\mathrm{nK}-\mathrm{m}^{2} / \mathrm{W}\right)$ & 9.24 & 8.87 & 0.49 \\
\hline \hline
\end{tabular}

one [Fig. 4(a)]. Even when metal and semiconductor are in ideal thermal contact $\left(r_{\mathrm{ms} \text {,actual }} \equiv 0\right)$, a nonzero resistivity $r_{\mathrm{LB}}$ is perceived. We call this the Lévy barrier effect, as this incorrectly perceived resistivity can be directly associated with the quasiballistic energy concentration and heat flux suppression near the semiconductor surface. These effects also have direct implications for the thermal performance of nanoscale electronic and optoelectronic devices. The Lévy barrier resistivity $r_{\mathrm{LB}}$ gets systematically higher as superdiffusive modes deviate more strongly from Brownian dynamics (smaller $\alpha$ ) and/or persist over longer distances (larger $u_{\mathrm{BD}}$ ) [Fig. 4(b)].

Experimental evidence for these important tendencies becomes clear upon comparing identification results for different materials (Table I). Semiconductor alloys, combining short penetration lengths (low diffusivity) with long MFPs (Rayleigh scattering), can be expected to exhibit considerable nondiffusive effects. Conventional characterization of InGaAs and $\mathrm{SiGe}$ indeed shows a strong frequency dependence of effective conductivity and severe Lévy barrier distortion on $r_{\mathrm{ms}}$ identification. Ballistic effects in single crystals such as GaAs, on the other hand, are almost negligible over the experimental range, and Fourier and TL characterizations match within a few percent.

It is remarkable that metal/semiconductor interfaces are found to be up to three times more conductive than currently believed. This trend persists at cryogenic temperatures (Fig. 5).

Although a close match between theory and experiments as for $\mathrm{Al} / \mathrm{Si}$ [Fig. 5(a)] has not yet been achieved, the $\mathrm{Al} / \mathrm{InGaAs}$ resistivities we obtained with our new approach are much closer to AMM and DMM model predictions than their conventionally extracted counterparts [Fig. 5(b)]. It is noteworthy that the remaining discrepancy of $3-4 \mathrm{nK}-\mathrm{m}^{2} / \mathrm{W}$ is well within reach of two correction terms that have already been investigated $[9,24]$. First, electron-phonon coupling in the aluminium was found to manifest itself as an additional series resistivity of $\simeq 1 \mathrm{nK}-\mathrm{m}^{2} / \mathrm{W}$ [24]. Second, the surface condition of the real-world metal/semiconductor interface deviates from the idealized one assumed within the AMM model. Studies have shown that a 3- to 5-nm imperfection layer gives rise to an additional resistivity of $\simeq 2-3 \mathrm{nK}-\mathrm{m}^{2} / \mathrm{W}$ [9]. Interestingly, these effects were deemed relatively unimportant in the original
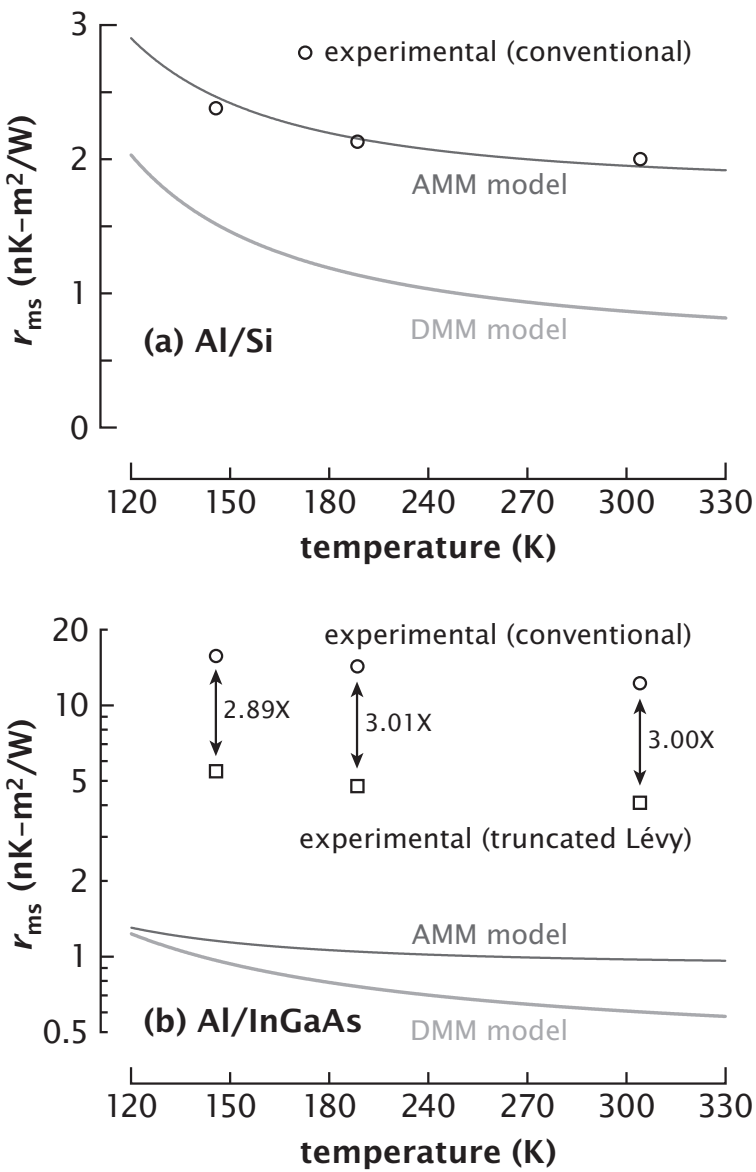

FIG. 5. Theoretical and experimental interface resistivity versus temperature. (a) Conventional identification of $\mathrm{Al} / \mathrm{Si}$, which does not exhibit quasiballistic effects over the measurement frequency range, shows good agreement with theoretical predictions. (b) Truncated Lévy identification of $\mathrm{Al} / \mathrm{InGaAs}$ brings the measured interface resistivity much closer to model predictions than conventional Fourier analysis. The remaining discrepancy between theory and experiment (3-4 nK- $\mathrm{m}^{2} / \mathrm{K}$ ) is well within reach of correction terms associated with electron-phonon coupling in the metal and atomic roughness of the interface.

publications, as they represented only a modest fraction of the then available Fourier-based experimental resistivity values. Our finding that the experimental resistivity is significantly lower than believed thus far puts these earlier efforts in a much more optimistic perspective. Adding both correction terms to DMM or AMM model predictions for an ideal Al/InGaAs interface yields theoretical estimations of $4-5 \mathrm{nK}-\mathrm{m}^{2} / \mathrm{W}$, in near-perfect agreement with our experimental TL results.

In summary, we have demonstrated the importance of properly accounting for ballistic heat effects in thermal interface metrology. Our results indicate that conventional Fourier analyses severely overestimate metal/semiconductor interface resistances due to misinterpretation of a superdiffusive heat barrier near the semiconductor surface. Decoupling interfacial dynamics from quasiballistic heat flow can be achieved by a TL formalism, providing opportunities for more accurate thermal characterization. Contrary to conventional approaches, our method yields experimental interface 
resistivities that are independent of laser modulation frequency, as physically appropriate, and that closely match theoretical predictions.

The InGaAs sample was provided by Hong Lu and Art Gossard. The SiGe sample was courtesy of Ed Croke. A.S. acknowledges funding from the Center for Energy Efficient
Materials, an Energy Frontier Research Center funded by the US Department of Energy, Office of Basic Energy Sciences, under Award No. DE-SC0001009, which supported the work of B.V. and Y.K; DARPA/Army Research Office Contract No. W911NF0810347, supporting the work of G.P. and A.M.; and AFOSR MURI Grant No. FA9550-08-1-0340 for partial support of experimental capabilities.
[1] P. L. Kapitza, J. Phys. (USSR) 4, 181 (1941).

[2] M. D. Losego and D. G. Cahill, Nature Mater. 12, 382 (2013).

[3] E. T. Swartz and R. O. Pohl, Rev. Mod. Phys. 61, 605 (1989).

[4] P. E. Phelan, ASME J. Heat Transf. 120, 37 (1998).

[5] E. Pop, S. Sinha, and K. E. Goodson, Proc. IEEE 94, 1587 (2006).

[6] D. G. Cahill, W. K. Ford, K. E. Goodson, G. D. Mahan, A. Majumdar, H. J. Maris, R. Merlin, and S. R. Phillpot, J. Appl. Phys. 93, 793 (2003).

[7] D. G. Cahill, K. Goodson, and A. Majumdar, ASME J. Heat Transf. 124, 223 (2002).

[8] R. M. Costescu, M. A. Wall, and D. G. Cahill, Phys. Rev. B 67, 054302 (2003).

[9] R. J. Stevens, A. N. Smith, and P. M. Norris, ASME J. Heat Transf. 127, 315 (2005).

[10] Y. K. Koh and D. G. Cahill, Phys. Rev. B 76, 075207 (2007).

[11] A. J. Minnich, J. A. Johnson, A. J. Schmidt, K. Esfarjani, M. S. Dresselhaus, K. A. Nelson, and G. Chen, Phys. Rev. Lett. 107, 095901 (2011).

[12] M. E. Siemens, Q. Li, R. Yang, K. A. Nelson, E. H. Anderson, M. M. Murnane, and H. C. Kapteyn, Nature Mater. 9, 26 (2010).

[13] K. T. Regner, D. P. Sellan, Z. Su, C. H. Amon, A. J. H. McGaughey, and J. A. Malen, Nat. Commun. 4, 1640 (2013).
[14] D. G. Cahill, Rev. Sci. Instrum. 75, 5119 (2004).

[15] J. A. Johnson, A. A. Maznev, J. Cuffe, J. K. Eliason, A. J. Minnich, T. Kehoe, C. M. Sotomayor Torres, G. Chen, and K. A. Nelson, Phys. Rev. Lett. 110, 025901 (2013).

[16] C. Bera, N. Mingo, and S. Volz, Phys. Rev. Lett. 104, 115502 (2010).

[17] T. Luo and G. Chen, Phys. Chem. Chem. Phys. 15, 3389 (2013).

[18] Z. J. Ziman, Electrons \& Phonons: The Theory of Transport Phenomena in Solids (Oxford University Press, New York, 2001).

[19] B. Vermeersch, A. M. S. Mohammed, G. Pernot, Y. R. Koh, and A. Shakouri, arXiv:1406.7342.

[20] See Supplemental Material at http://link.aps.org/supplemental/ 10.1103/PhysRevB.90.014306 for Suppl. Fig. S1.

[21] Y. K. Koh, S. L. Singer, W. Kim, J. M. O. Zide, H. Lu, D. G. Cahill, A. Majumdar, and A. C Gossard, J. Appl. Phys. 105, 054303 (2009).

[22] A. A. Maznev, J. A. Johnson, and K. A. Nelson, Phys. Rev. B 84, 195206 (2011).

[23] R. Metzler and J. Klafter, Phys. Rep. 339, 1 (2000).

[24] P. Singh, M. Seong, and S. Sinha, Appl. Phys. Lett. 102, 181906 (2013). 